\title{
Limitations of Imaging, Medico-Legal Issues and the Importance of Published Medical Literature
}

\section{Sohoni CA}

Medicos clinic, Koregaon Park, Pune, Maharashtra, India

\section{Introduction}

With increasing awareness about disease prevention and with corporate hospitals and diagnostic centers aggressively marketing health check-up packages, a significant number of apparently healthy people undergo ultrasound examination. More often than not such studies are seen just as a formality. However, these apparently simple studies can sometimes be very tricky. Two such cases are illustrated here.

\section{Case 1}

A 34 year old asymptomatic young adult male presented himself for an ultrasound examination of abdomen and pelvis as a part of routine health check-up. The study was almost concluded as normal when a subtle, ill-defined hypoechoic area was seen in the left renal sinus. There was no focal bulge of the overlying renal cortex and no obvious anatomical distortion of adjacent renal parenchyma. Even colour Doppler examination did not show any significantly increased vascularity or obvious displacement of vessels in this region. Since the patient was also undergoing a CT Coronary angiography as a part of the

Correspondence to: Dr. Chandrashekhar A. Sohoni, Consultant Radiologist, B-5, Common Wealth Hsg. Soc., Opp. Bund Garden, Pune-411001, Maharashtra, India. E-mail:- sohonica@gmail.com

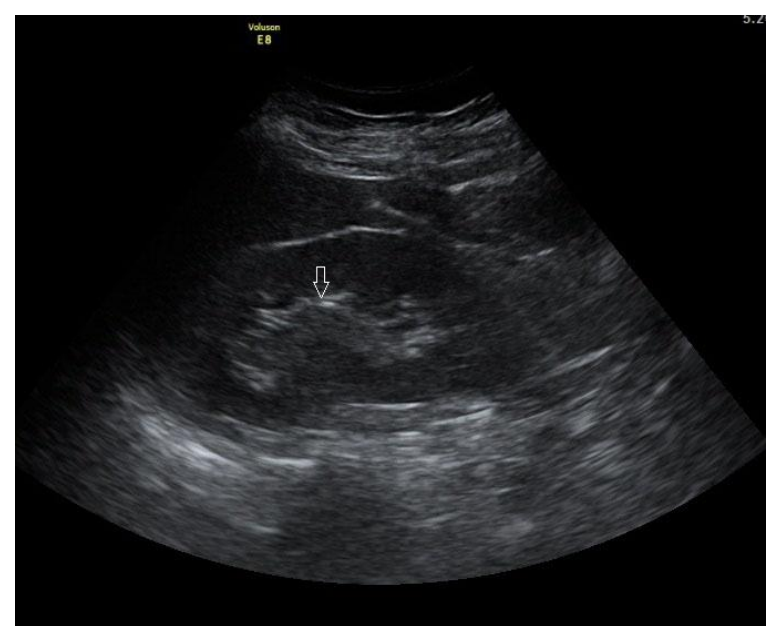

Fig. 1a: The ultrasound image of left kidney shows an area of subtle hypoechogenicity in the renal sinus.

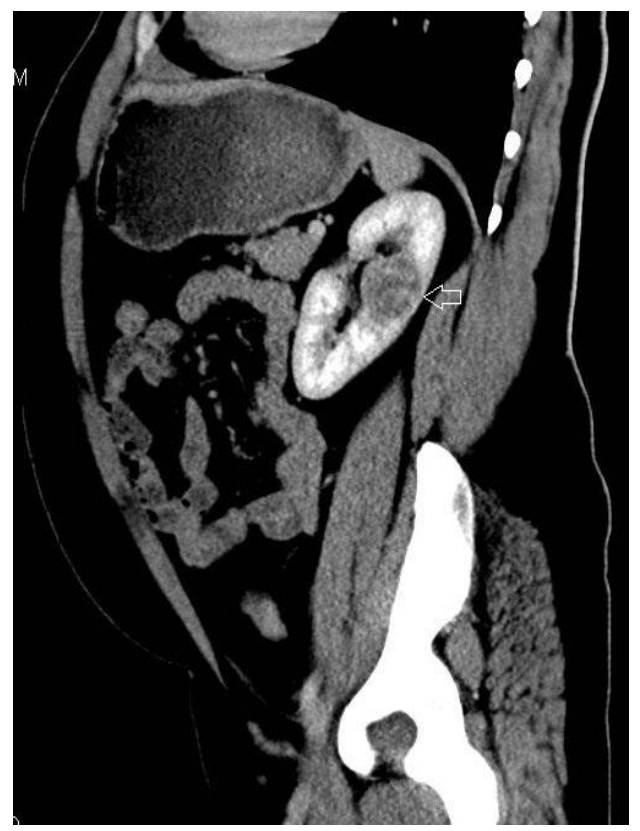

Fig. 1b: The CT study reveals a focal, mildly enhancing mass lesion suggestive of a neoplastic etiology. 
package, the scan was extended to cover the upper abdomen in order to screen the left kidney. The subtle area of suspicion in the left renal sinus which appeared so inconspicuous on ultrasound turned-out to be a mildly enhancing mass lesion measuring $3.9 \times 3.8 \mathrm{~cm}$, likely to represent a neoplastic lesion.

\section{Case 2}

A 33 year old young adult male with a past history of ureteroscopic removal of right ureteric calculus 6 years ago came for an ultrasound examination as a part of routine health check-up. The patient was asymptomatic at present. This was his first ultrasound examination in five years. Ultrasound revealed a round echogenicity measuring $13 \mathrm{~mm}$ with subtle posterior acoustic shadowing, seen in the lower calyx of left kidney. It was clearly suggestive of a calculus. However, radiograph of the lumbar spine performed later in the day as a part of health check-up did not show any corresponding radio-opacity. A screening CT study did not reveal any calculus in the left kidney. The echogenic area mimicking a stone on ultrasound was likely to be focal renal sinus fat.

Apparently there is nothing unusual in the above cases because false negative and false positive results are well known with imaging modalities, particularly radiography and ultrasonography. However, these limitations of imaging can lead to errors which in-turn can lead to medico-legal issues. Irrespective of their level of experience and expertise, most radiologists would agree of having faced the brunt of imaging errors in one way or the other.

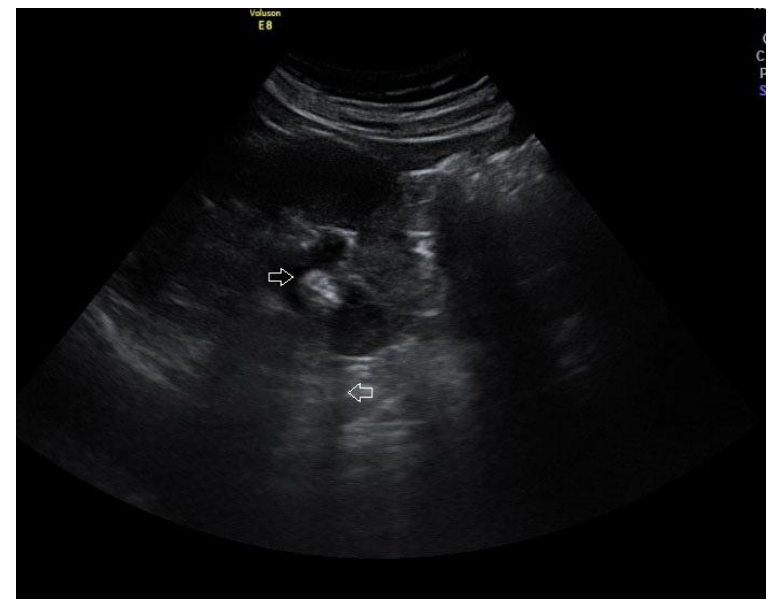

Fig. 2a: The ultrasound image shows an echogenicity in the lower pole of left kidney with subtle posterior acoustic shadowing, suggestive of a calculus.

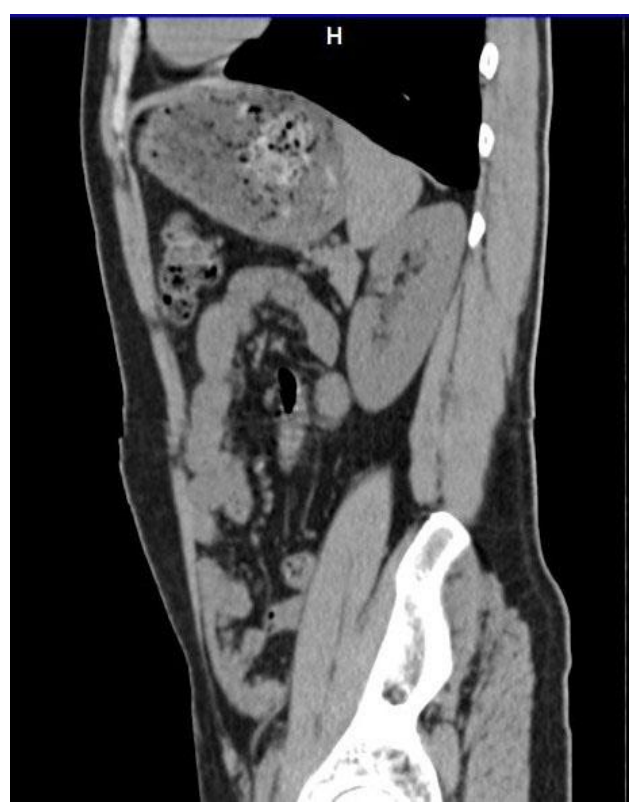

Fig. 2b: The CT study however did not reveal any calculus.

People undergo health check-ups so that diseases get diagnosed before they become clinically manifest. Hence, missing a tumor in early stage would be exactly contradictory to the basic tenet of preventive health checkup. Due to absence of symptoms, such missed lesions are likely to go undetected till late. Since missing a lesion in pre-clinical stage can have a direct bearing upon the final prognosis, it may invoke medico-legal 
issues. Prima facie, an aggrieved patient appears clearly justified in suing a radiologist on this ground, although many other factors would be considered by the court of law before passing a verdict. A false positive diagnosis can also be problematic, particularly if it leads to an unwarranted surgery. To the patient, the radiologist appears as a culprit in the temporal sequence of such events.

While some facts likes operator dependability, false positive and false negative results with imaging modalities are well established medical facts, radiologists still find it difficult to explain themselves when an important radiological finding is missed. Unlike complex clinical issues, a missed radiological finding is a very apparent error and hence easier for nonmedical people to understand. To a common man who doesn't understand the intricacies of imaging science, a missed radiological finding appears as a clear act of omission on the part of radiologist. Even if the limitations and fallacies of imaging are mentioned in medical literature, that doesn't stop an aggrieved patient from challenging a radiologist in the court of law.

Published medical literature can have a bearing on court verdicts, because such literature helps the court of law to gauge the average standard of care and thus decide whether there has been a significant deviation from the standard practice. ${ }^{3}$

As regards Case 1, some studies have suggested that ultrasound may have a problem detecting renal masses that are less than $3 \mathrm{~cm} .{ }^{1}$ For renal mass lesions which are 25-30 mm, studies have reported the detection rate of ultrasound to be as good as
$100 \% .^{2}$ Thus, looking at the size of the lesion in our case, missing it on ultrasound would look like a gross blunder when compared with currently available medical literature. However, the factors affecting diagnostic accuracy of imaging are:-

a.The level of suspicion

b.The difference in radiologic appearance between normal tissue and abnormal tissue

c. Variable human perception.

The reason for the "almost missed" renal lesion in Case 1 was the low level of suspicion, the subtle hypoechogenicity of the lesion as compared to surrounding tissue and lack of anatomical disfigurement; whereas in Case 2, the renal sinus fat masquerading as a calculus of that size is extremely unusual. But these factors which lead to errors in diagnosis vary from case to case and hence usually go unreported.

Isolated errors in imaging find it difficult to get published, firstly because imaging diagnosis is subjective and secondly because isolated errors lack statistical significance from the standpoint of publication. Having said that one cannot refute the fact that certain diagnostic pitfalls that are well known in radiology circles. Missing a liver abscess, gall bladder calculi, renal calculi and even renal masses are some of the common embarrassing experiences amongst radiologists.

It is important to report fallacies of imaging from time to time, because they keep occurring despite improvement in imaging machinery. The simple fact that a particular type of error is known to occur and has been reported previously goes a long way in 
helping a professional, particularly in cases of alleged medical negligence.

The usual tendency is to sweep errors under the carpet while glorifying achievements. This tendency can backfire, especially in an era where patients are extremely inquisitive and not shy to invoke their legal rights. Without supporting medical literature radiologists may find it difficult to defend themselves in front of non-medical people including the court of law.

While one cannot deny the fact that doctors are sometimes negligent of their duties, one also cannot deny that many more doctors suffer undeservedly in medico-legal cases. Hence, for the betterment of the fraternity, it is important to give medical publishing the new dimension of legality. The idea is not to avert responsibility but to be honest about capabilities and limitations.

\section{References}

1. Einstein DM, Herts BR, Weaver R, Obuchowski N, Zepp R, Singer A. Evaluation of renal masses detected by excretory urography: cost-effectiveness of sonography versus CT. AJR 1995;164(2):371-375.

2. Jamis-Dow CA, Choyke PL, Jennings SB, Linehan WM, Thakore KN, Walther MM. Small $(<$ or $=3 \mathrm{~cm})$ renal masses: detection with $\mathrm{CT}$ versus US and pathologic correlation. Radiology 1996;198(3):785-8.

3. Radiology Medico-legal cases. Indian J Radiol Imaging 2008;18:117-8. 